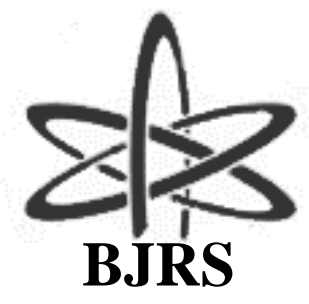

\author{
BRAZILIAN JOURNAL \\ OF \\ RADIATION SCIENCES \\ 09-01A (2021) 01-08
}

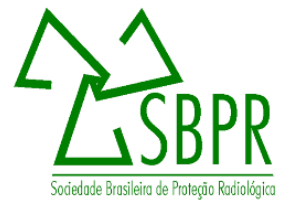

\title{
Efficiency stability of HPGe detectors under distinct count rates
}

\author{
Zahn G. S., Genezini F. A. \\ Instituto de Pesquisas Energéticas e Nucleares (IPEN-CNEN/SP) \\ Av. Professor Lineu Prestes 2242 \\ 05508-000 São Paulo, SP, Brazil \\ gzahn@ipen.br
}

\begin{abstract}
In this work the reproducibility of the efficiency of different HPGe detectors was analyzed under a series of different conditions. The detectors studied were plugged either to a regular analogical amplifier or to a digital signal processing (DSP) device, to evaluate the possible differences between either setup. Detectors were inspected by performing a long series of sequential measurements with standard calibration sources and comparing the standard deviation of the number of counts per second in each series to the uncertainty of the individual measurements. Detectors were also subjected to distinct count rates, to verify the possible experimental issues associated with this parameter. The results allow a discussion on the stability of the detectors' efficiencies over a few days, the possible dependence with the count rate, and the estimation of the uncertainty related to the efficiency variation.
\end{abstract}

Keywords:HPGe, efficiency stability, pile up. 


\section{INTRODUCTION}

Nuclear application often require - and confidently assume - that the efficiency of a radiation detector is constant during the measurement series, which can span for a few days or even weeks [1]. Comparative Neutron Activation Analysis, for example, assumes the detector efficiency to be constant over the process of counting all standards and samples, which usually takes 2-3 days, but may take more time in some cases - this assumption enables the technique to disregard the detector efficiency as a source of uncertainty [2-4].

High Purity Germanium (HPGe) detectors are by far the most used detectors in high resolution gamma spectrometry due to their excellent energy resolution (usually around 1-3 keV for the 100-2000 keV range) [5]. An important feature of these detectors is that the semiconductor crystal operating bias is always defined above the voltage required to fully deplete the crystal, in a way that small variations in the bias voltage will not change the sensitive volume of the detector. Moreover, while the operating bias is usually quite high $(1500-5000 \mathrm{~V})$, the current is very low, in the range of $\mathrm{pA}$, so the power dissipation is very low and that fact, together with the constant refrigeration of the crystal and part of the preamplifier (usually accomplished by thermal contact with a liquid nitrogen container), keeps the detector at a very stable temperature. These two facts contribute to a remarkable stability in the efficiency and gain of HPGe detectors.

It is known from practical experience that the gain of an HPGe detector can oscillate slowly and slightly over short periods, usually less than $0.1 \%$ if the system is in good shape - in practical terms, this means that in a given spectrum, a gamma-ray peak may be found at energies up to $1 \mathrm{keV}$ away from the expected value. On the other hand, while there are a few studies on their long-term efficiency stability [6-9], and a lot of studies on the quantification of secondary detection effects $[10,11]$, there are very few experimental data on the short-term stability of the HPGe detectors' efficiency or in the dependence of this stability with the count rate.

In this work, an experimental verification of the efficiency stability of two HPGe detectors was performed in order to check the limits of this stability and its dependence with the count rate or with the data acquisition setup used. 


\section{EXPERIMENTAL PROCEDURE}

In order to asses the possible variation of the detection efficiency with time, a radioactive source of ${ }^{60} \mathrm{Co}$ was counted 100 times sequentially (LiveTime $=2400 \mathrm{~s}$ ) without any source manipulation between measurements, to avoid the possibility of changes in the detection geometry. The spectra were then analyzed using Canberra's Genie-2000 software, and the variation in the count rates observed in the $1173 \mathrm{keV}$ peak was studied by determining the standard deviation $(S D)$ of the obtained values and comparing it to the average uncertainty $(1 \sigma)$ of the peak areas obtained in the individual acquisitions. This way, if the efficiency is constant, all variation should arise from counting statistics alone, so that $S D=\sigma$; on the other hand, if the efficiency varies between measurements, the observed standard deviation $S D$ will be greater than the uncertainty (i.e., $S D>\sigma$ ), due to the variation in the counting efficiency. To quantify this variation, two distinct variables were proposed, the relative uncertainty increase (RUI, Eq. 1), which is an indication of the how relevant this variation is compared to the intrinsic counting uncertainty, and the absolute uncertainty increase (AUI, defined in Eq. 2), which indicates the uncertainty value that should be added in the propagation should the detector be used at that count rate.

$$
\begin{aligned}
& R U I(\%)=100 \times \frac{S D-\sigma}{\sigma} \\
& A U I(\%)=S D(\%)-\sigma(\%)
\end{aligned}
$$

As this efficiency variation could be dependent on the count rate, this process was repeated using the same radioactive source at several different source-detector distances, in order to obtain distinct count rates. Also, in order to check for possible differences depending on the digitalization process, the measurements were repeated with two similar HPGe detectors coupled to distinct signal processing chains - in all cases, spectra were acquired with 8192 channels and the Pile-Up Reject (PUR) guard was turned off: 
- C3: Canberra GX2020, coupled to a conventional analog amplifier (Canberra 1510, 4 $\mu$ s shaping time) and to a regular MCA acquisition board (Canberra S-100) that digitizes the amplifier output;

- C5: Canberra GC2018, coupled to a Canberra DSA-1000 Digital System Processing (DSP) unit that directly digitizes the detector's preamplifier output without using an analog amplifier - the trapezoidal filter settings are rise time $=5.6 \mu$ s, flat top $=2.0 \mu$ s.

\section{RESULTS AND DISCUSSION}

The first step was to check the dependence of the dead time, informed by the acquisition system, with the count rate - to simplify the matter only the count rate for the $1173 \mathrm{keV}$ peak was used. These results, shown in Fig. 1 , indicate that in the interval observed in this study the dead time increases linearly with the count rate; moreover, they clearly show that the DSP system (C5) is much more robust at high count rates, presenting a much lower dead time than the analog system (C3) at the same count rate. This difference indicates that the DSP system processes the detector signal in a rather shorter time than the analog amplifier with $4 \mu s$ shaping time.

Figure 1:Dependence of the acquisition dead time with the count rate observed in the $1173 \mathrm{keV}$ peak from ${ }^{60}$ Co for the two detector systems studied.

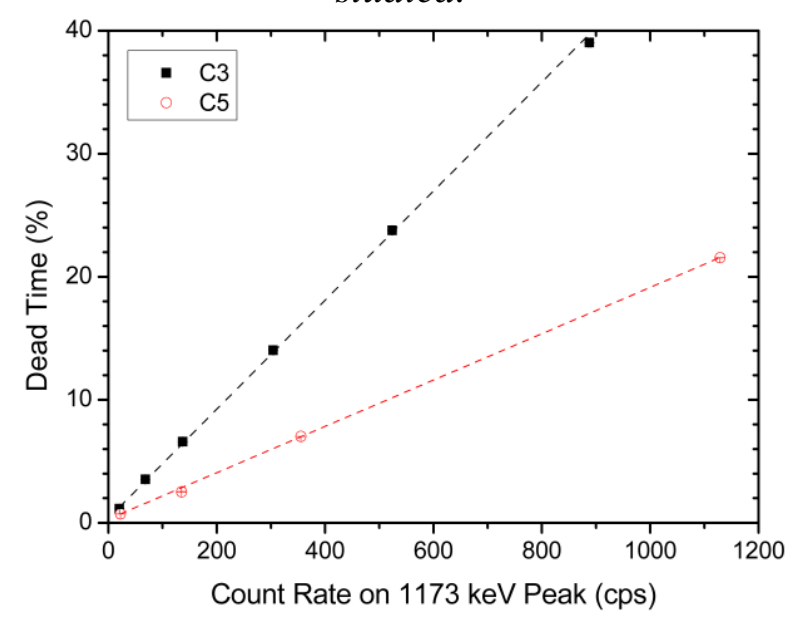


The results for the absolute uncertainty increase (AUI) and relative uncertainty increase (RUI) as a function of the count rate observed for the $1173 \mathrm{keV}$ peak are presented in Fig. 2, and in both cases it is clear that the two detectors show an identical behavior.

Figure 2:Dependence of the relative (left) and absolute (right) uncertainty increase with the count rate observed in the $1173 \mathrm{keV}$ peak from ${ }^{60} \mathrm{Co}$ for the two detector systems studied.
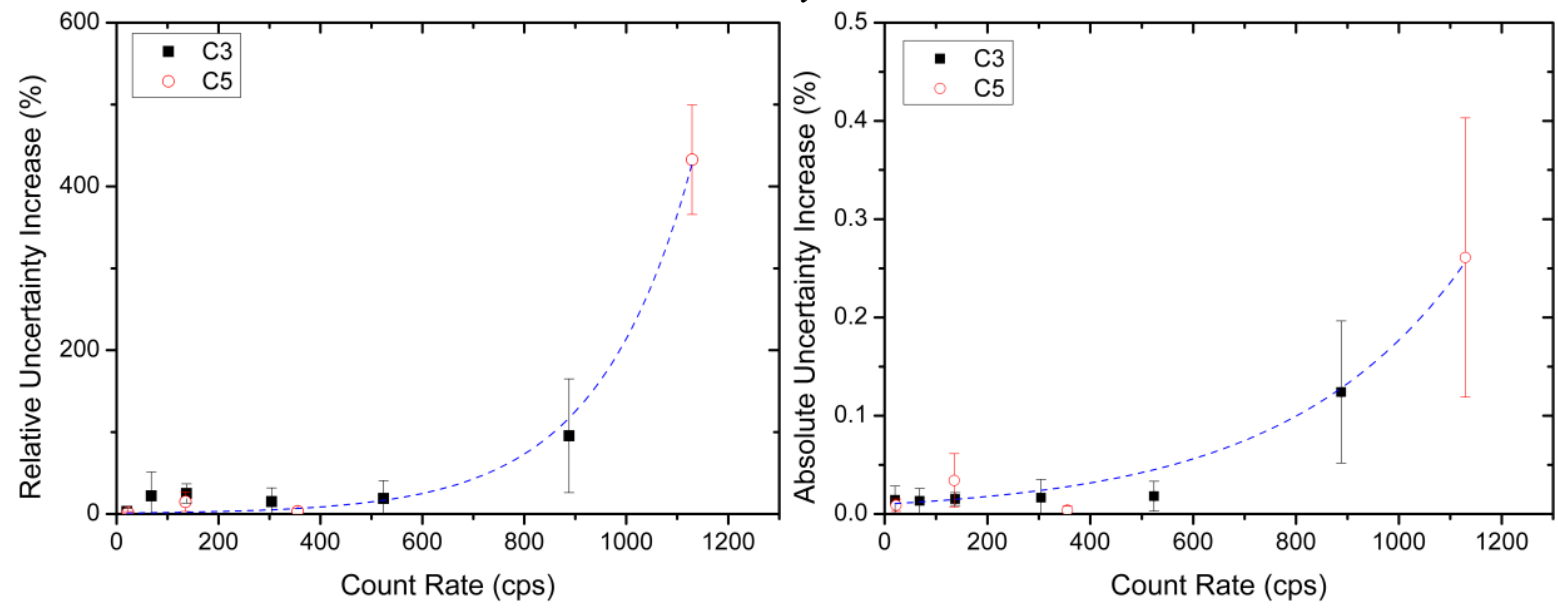

Analyzing the results in the left side of Fig. 2 it is clear that at low count rates the efficiency is very stable, with all the count rate variation originating in the statistical oscillation of the counts; however, for count rates above $\approx 700-800 \mathrm{cps}$ there is indeed a relevant increase in the efficiency oscillation. Looking at the right side of Fig. 2, however, it is possible to infer that, at least for those count rates, the influence of the efficiency oscillation at high count rates will contribute at most with $0.3 \%$ to the final measurement uncertainty, which in most cases is a negligible amount.

\section{CONCLUSION}

The results obtained in this work show that for the same count rate the detector coupled to a Digital Signal Processing unit presented much lower dead times than the one connected to a conventional amplifier-MCA system, possibly due to the great difference in the form the detector 
signals are processed - further tests with other analog amplifiers and digitizers are required to check this assumption, though.

As for the efficiency stability, in the usual operational conditions, where dead times should be kept below $10 \%$, both detectors were very stable, with a negligible increase in the count rate fluctuations; at higher count rates, though, large fluctuations were observed which seem related to the count rate and not to the dead time. The reasons for this increase in the count rate fluctuation have to be studied, but may be related to pile-up fluctuations or to peak fit issues, as at higher count rates the detector's resolution in known to worsen. Nevertheless, in these tests, this fluctuation would require at most a $0.3 \%$ increase in the measurement uncertainty.

Finally, it must be stressed that these tests were performed with a standard ${ }^{60} \mathrm{Co}$ source, whose peaks are intense and very clearly separated; further tests have to be performed in order to check how these fluctuations will interfere in the results of weaker peaks in more complex spectra. 


\section{REFERENCES}

[1] COLLINS, S.M.; HARMS, A.V.; REGAN, P.H. Half-life determination of the ground state decay of ${ }^{111}$ Ag, Appl Radiat Isot, v.108, p. 143-147,2016.

[2] KUCERA, J.; BODE, P.; STEPANEK, V. The 1993 ISO Guide to the Expression of Uncertainty in Measurement Applied to NAA, J Radioanal Nucl Ch, v. 245, p. 115-122, 2000.

[3] BALLA, M. ; MOLNAR, Z., KOROS, Á Uncertainty Budget and Validation of NAA Using Reference Materials, J Radioanal Nucl Ch, v. 259, p. 395-400, 2004.

[4] ZAHN, G. S. ; TICIANELLI, R. B. ; LANGE, C. N. ; FAVARO, D. I. T. ; FIGUEIREDO, A. M. G. Uncertainty Analysis In Comparative NAA Applied To Geological And Biological Matrices, In: INTERNATIONAL NUCLEAR ATLANTIC CONFERENCE, 2015, São Paulo, Annals... São Paulo, Comissão Nacional de Energia Nuclear, 2015.

[5] LOVElAnD, W. D. ; MORRISSEY, D. J. ;SEABORG, G. T., Modern Nuclear Chemistry, Hoboken : John Wiley \& Sons, 2006.

[6] SIEGERT, H; SCHRADER, H. ;SCHÖTZIG, U. Half-life measurements of Europium radionuclides and the long-term stability of detectors, Appl Radiat Isot, v.49, p. 13971401,1998 .

[7] AlveS, A. P. ; AMARAl, A. M., KASTnER, G. F. ;FERREIRA, A. V. HPGe Gamma Detector:Efficiency Curve Evaluationduring Ten Years Of Operation, In: INTERNATIONAL NUCLEAR ATLANTIC CONFERENCE, 2009, Rio de Janeiro, Annals... Rio de Janeiro, Comissão Nacional de Energia Nuclear, 2009.

[8] ZAHN , G. S. ; GENEZINI, F. A. ; TICIANELLI, R. B. ; SAIKI, M. Long-term performance assessment of HPGE detectors used in the neutron activation analysis laboratory of IPENCNEN/SP (Brazil), Appl Radiat Isot, v125, p. 108-112,2017.

[9] SZYMANSKA, K et al Resolution, efficiency and stability of HPGe detector operating in a magnetic field at various gamma-ray energies, Nucl. Instrum. Methods. Phys. Res. A, v. 592, p. 486-492, 2008.

[10]CASTRO, R. M. et al Efficiency Loss in HPGe Detectors due to Beta and Gamma Sum Coincidence, Braz J Phys, v. 35, p. 754-756, 2005. 
[11]BECKER, J. A et al Precision Measurements Of Nuclide Half-Lives, Nucl. Instrum. Meth, v. 155, p. 211-220, 1978. 